\title{
Erratum: Synchrotron oscillation effects on an rf-solenoid spin resonance [Phys. Rev. ST Accel. Beams 15, 124202 (2012)]
}

\author{
P. Benati, D. Chiladze, J. Dietrich, M. Gaisser, R. Gebel, G. Guidoboni, V. Hejny, A. Kacharava, \\ V. Kamerdzhiev, P. Kulessa, A. Lehrach, P. Lenisa, B. Lorentz, R. Maier, D. Mchedlishvili, W. M. Morse, \\ D. Öllers, A. Pesce, A. Polyanskiy, D. Prasuhn, F. Rathmann, Y. K. Semertzidis, E. J. Stephenson, \\ H. Stockhorst, H. Ströher, R. Talman, Yu. Valdau, Ch. Weidemann, and P. Wüstner \\ (Received 8 February 2013; published 2 April 2013)
}

DOI: 10.1103/PhysRevSTAB.16.049901

PACS numbers: 29.27.Bd, 29.20.db, 29.27.Hj, 41.75.Ak, 99.10.Cd

There is a sign error in Eq. (1) that should be changed to read

$$
\vec{\omega}=-\frac{q}{m}\left\{G \vec{B}-\left[G-\left(\frac{m}{p}\right)^{2}\right] \frac{\vec{\beta} \times \vec{E}}{c}\right\} .
$$

This equation is a part of the Introduction and is intended only to illustrate the two methods for obtaining a "frozen" spinpolarized beam where the polarization and the velocity are parallel. It is restricted to the case where the particle travels along the central orbit in a storage ring in a direction perpendicular to the radial and vertical directions. Given that these are also the directions of the magnetic and electric fields, this means that $\vec{\beta} \cdot \vec{B}=\vec{\beta} \cdot \vec{E}=0$. A more complete treatment of the precession of the spin in electric and magnetic fields is given by the Thomas-Bargmann-Michel-Telegdi equation, which is described in Ref. [1].

Equations (8) and (9) should be combined in order to make it clear how the time dependence is applied:

$$
\theta_{S}=2 \pi \epsilon \cos \left(2 \pi f_{\mathrm{CYC}}(1-G \gamma) t+\delta(t)\right) .
$$

In both Eqs. (8) and (15) there should be an additional phase included in the argument of the cosine function. This phase ranges from 0 to $2 \pi$ and should be chosen randomly. Such a phase was included and found to have negligible effects on any polarization phenomena. This arises in part because the time scale for synchrotron oscillations is orders of magnitude from either betatron oscillations or the sign changes due to the operation of the rf solenoid, as shown in Fig. [9]. It was thus not included in the paper.

The caption to Fig. 8 states that the ramp speed for the Froissart-Stora frequency scan is $40 \mathrm{~Hz} / \mathrm{s}$ when it should really be $10 \mathrm{~Hz} / \mathrm{s}$, in agreement with the text.

None of these changes have any bearing on the main conclusions of this paper.

\section{ACKNOWLEDGMENTS}

The authors wish to acknowledge S. Mane for bringing our attention to some of these corrections.

[1] J. D. Jackson, Classical Electrodynamics (Wiley, New York, 1998), 3rd ed.

Published by the American Physical Society under the terms of the Creative Commons Attribution 3.0 License. Further distribution of this work must maintain attribution to the author(s) and the published article's title, journal citation, and DOI. 